\title{
Brief interventions for alcohol misuse
}

\author{
Anne Moyer PhD, John W. Finney PhD \\ See also page 479 and www.cmaj.ca/lookup/doi/10.1503/cmaj.140849
}

Competing interests: None declared.

This article has been peer reviewed.

Correspondence to: Anne Moyer, anne.moyer @stonybrook.edu

CMAJ 2015. DOI:10.1503 /cmaj.140254
A $s$ the burden of alcohol misuse - in terms of disease, trauma and social problems - grows in Canada, increased access to brief interventions is recommended. Such access forms part of a comprehensive two-tiered response involving population-level changes, such as regulating the marketing, pricing and availability of alcohol, and more focused practices, such as education and intervention. ${ }^{1}$ In 2007, recommendations from Canada's National Alcohol Strategy Working Group identified screening, brief intervention and referral to specialist treatment as an important strategy for reducing the negative health effects of excessive alcohol consumption, particularly injury and chronic disease. ${ }^{2}$ Here, we discuss a practical approach to the delivery of brief interventions based on evidence supporting their effectiveness (Box 1). This approach is intended for use by health care providers who determine, during the provision of primary, specialty or hospital care, that an individual is engaging in unhealthy drinking.

\section{What is the purpose of a brief intervention for alcohol misuse?}

A common goal of brief interventions for alcohol misuse is to reduce drinking to recommended low-risk patterns and levels, not necessarily to urge abstinence. The intent is to reduce overall consumption and harmful practices like binge drinking, so as to lower the likelihood of such negative health and social consequences as chronic disease (liver disease, stroke, cancer, diabetes mellitus), damage to a fetus, motor vehicle crashes, injuries, domestic violence, family discord, and occupational or academic instability.

For healthy adults up to 65 years of age, Canadian guidelines for low-risk alcohol drinking advise consuming no more than 10 drinks a week for women (with no more than 2 drinks a day on most days) and no more than 15 drinks a week for men (with no more than 3 drinks a day on most days). ${ }^{3}$ In addition, the guidelines recommend not drinking when engaging in potentially dangerous activities, minimizing intoxication, abstaining from alcohol when pregnant or intending to become pregnant, and delaying the uptake of drinking by youth. These guidelines were developed from evidence supporting the levels and patterns of alcohol consumption that would reasonably minimize health risks, negative social consequences and prenatal harm. ${ }^{4}$

A health care provider can undertake brief interventions during routine health care visits or during a hospital stay for a different condition. In addition, focused screening is useful in contexts where problematic alcohol use is common (e.g., emergency departments) or in groups for whom it is particularly harmful (e.g., obstetrics patients or people who have been arrested for driving while intoxicated). ${ }^{5}$ Typically, brief interventions are considered most suitable for those whose

\section{Box 1: Evidence used in this review}

We searched PubMed and MEDLINE databases using combinations of the terms "brief," "intervention," "alcohol" and "review" along with the "related articles" function. These searches yielded citations numbering in the hundreds; we focused our attention on the most recently published research, particularly state-ofthe-art systematic reviews and a recent systematic review of reviews. We selected the examples of brief interventions featured here to illustrate differing salient aspects of their content and delivery (intensiveness and intensity, type of practitioner) with a focus on high-quality research (i.e., randomized controlled trials) and evidence related to the Canadian context. 
drinking does not meet the criteria for an alcohol use disorder, but they may also be a valuable initial component of a more comprehensive or stepped-care form of treatment. ${ }^{6}$

\section{What constitutes a brief intervention?}

\section{Screening}

A brief intervention is usually preceded by screening - that is, asking targeted questions about drinking behaviour. The College of Family Physicians of Canada and the Canadian Centre on Substance Abuse have developed a protocol for screening, brief intervention and referral based on three screening questions (Box 2). ${ }^{7}$ Other commonly used screening tools include the Alcohol Use Disorders Identification Test Consumption Items (AUDIT-C) ${ }^{12}$ and the CAGE questionnaire. ${ }^{13}$

The three-item AUDIT-C can be used to assess a range of severity of drinking problems, from risky drinking to alcohol use disorders. ${ }^{14} \mathrm{It}$ consists of the following three questions: How often have you had a drink containing alcohol in the last year? How many drinks containing alcohol did you have on a typical day when you were drinking in the last year? How often in the last year have you had six or more drinks on one occasion?

The four-item CAGE questionnaire asks about key areas that would indicate that drinking has become problematic: feeling the need to cut down, having been criticized for drinking, feeling guilty about drinking or feeling the need to drink first thing in the morning. The CAGE questionnaire is not recommended to be used alone (without additional questions to assess levels of recent consumption), because it does not identify people with elevated alcohol consumption who have not experienced problems related to their drinking and because it asks about lifetime experiences, so may generate a positive screening result for people who are no longer consuming alcohol or are drinking at low-risk levels. ${ }^{14}$ Computer-assisted screening and incorporation of screening into existing clinic information-gathering procedures can be effective in relieving provider burden. ${ }^{15,16}$

\section{Brief interventions}

When risky alcohol use is identified through screening, brief interventions, commonly involving individualized feedback and counselling, can then be initiated. The interventions available for use are diverse but typically involve giving brief advice and deliberately employing client-centred, nonconfrontational, motivational approaches to avoid creating resistance to change. ${ }^{17}$ The most commonly used elements in this kind of motivational interviewing have been summarized by the acronym FRAMES: Feedback of risk, encouraging Responsibility for change, Advice, a Menu of options, therapeutic Empathy and enhancing Self-efficacy. ${ }^{18}$ Providers help to set goals and suggest useful change strategies, which can often be bolstered by supplemental materials, such as pamphlets or workbooks.

The intensity of contact varies from very brief (less than 5 minutes, with no follow-up) through brief (less than 15 minutes, with no follow-up) to brief but repeated (individual sessions of less than 15 minutes, with multiple follow-up contacts). ${ }^{19}$ One example of a very brief intervention

Box 2: Description of the screening, brief intervention and referral protocol of the College of Family Physicians of Canada and the Canadian Centre on Substance Abuse ${ }^{7}$

The protocol begins with three screening questions. If the response to the first question ("Do you drink beer, wine, coolers or other alcoholic beverages?") is "no," reasons are explored (e.g., religion, family history, medications or being in recovery). If, for example, the patient is in recovery and reports doing well, abstinence is supported and reinforced. If the patient is in recovery and reports not doing well, then follow-up involves support, assessment for concurrent mental illness, and referral to further addiction treatment or community support resources, as needed.

If the patient answers "yes" to the first question, two additional questions are asked: "On average, how many days per week do you drink alcohol?" and "On a typical drinking day, how many drinks do you consume?"

If the patient's consumption is within the Canadian limits for low-risk drinking, the behaviour is reinforced, a copy of the low-risk drinking guidelines is provided, and screening is repeated annually. If the patient is part of a subgroup for which even lower levels of alcohol consumption are recommended (such as youth and young adults, pregnant or breastfeeding women, adults older than 65 years, people at risk for chronic conditions that are more likely to occur with or be exacerbated by alcohol consumption ${ }^{8}$ and those coping with prior alcohol dependence), then he or she is advised to reduce alcohol consumption and is referred to such resources as community support groups and addiction treatment services.

If the patient is drinking more than the low-risk limits, further assessment is used to determine if he or she meets the criteria for alcohol dependence or alcohol abuse set out in the American Psychiatric Association's Diagnostic and Statistical Manual of Mental Disorders, fourth revision (DSM-IV), ${ }^{9}$ or is simply at elevated risk. (The new version of this classification, DSM-5, ${ }^{10}$ uses a single category, alcohol use disorder, with mild, moderate and severe subclassifications; ${ }^{11}$ it has not yet been incorporated into the Canadian protocol.)

Brief intervention and follow-up protocols for each of three levels of risk (elevated risk, alcohol abuse or alcohol dependence) are provided. Each intervention begins with a session of advice and assistance during which the health care provider gives feedback on risk stage, assesses the patient's readiness to change and engages in nonjudgmental discussion. For those at elevated risk, cutting down to low levels of risk is encouraged; for those with alcohol abuse, cutting down or abstaining is suggested; and for those with alcohol dependence, abstinence with medication support is recommended. Depending on the patient's readiness to change and the level of risk, reiterating concern, providing support, assisting in goal planning, referring to community resources (such as Alcoholics Anonymous or formal provincial treatment services), monitoring for withdrawal and/or prescribing medication are offered. If drinking goals have not been met by the time of next follow-up, these types of assistance are provided again. 
consisted of just five minutes of advice (feedback of screening results and review of associated adverse health consequences) delivered by a nurse to patients aged 55 and older with hazardous drinking levels. ${ }^{20}$ An example of a brief multicontact intervention was directed at college students during routine visits with their primary care providers at five US and Canadian health clinics. ${ }^{21}$ A 15-minute intervention and a 15-minute reinforcement session with the physician were scheduled one month apart, followed by two telephone calls from a primary care interventionist. The sessions involved motivational interviewing techniques. Students were given a manual that included feedback about their drinking, descriptions of drinking norms and the adverse effects of drinking among college students, exploration of personal likes and dislikes related to drinking, worksheets on drinking cues, instruction on how to calculate blood alcohol levels, consideration of the effects of alcohol on life goals, strategies for cutting down, a drinking contract and drinking diary cards.

\section{Follow-up}

Follow-up involves additional assessment, reinforcement and, if necessary (i.e., if drinking becomes more hazardous), referral to more intensive treatment.

\section{How effective are brief interventions?}

Much investigation has been directed at establishing the effectiveness (real-world performance) of brief interventions for alcohol abuse

\section{Box 3: Applying the results of this review in clinical practice}

Brief interventions are best applied for persons who do not meet the criteria for an alcohol use disorder, especially one of high severity. However, if such a person declines referral to specialized treatment, a brief intervention, using motivational interviewing techniques, can be employed to generate more openness to specialized treatment or as a first step in a planned, stepped-care approach.

When employing a motivational brief intervention with a person who already is committed to change, it is better not to focus on the "pros" of drinking or substance use in a decisional balance component. Although focusing on the positive aspects of drinking or substance use has not yet been studied in relation to undermining an individual's commitment to change ${ }^{26}$ some evidence of this effect has been found with patients who use cocaine. ${ }^{27}$ For persons who are ambivalent about changing their drinking or substance use behaviour, exploring the decisional balance can be effective.

Simple referral to treatment or a mutual help group is often, by itself, ineffective. More effective are interventions that have been developed and can be applied to increase engagement. ${ }^{28-30}$ Furthermore, for patients with accompanying psychiatric or social conditions and more severe dependence, more intense monitoring and management of recovery following treatment may be necessary. ${ }^{31}$ in primary care. One recent comprehensive and rigorous systematic review of published systematic reviews provided an authoritative summary of this evidence..$^{22}$ Overall, across 24 systematic reviews (summarizing a total of 56 individual trials), brief alcohol interventions delivered in primary health care settings were effective in reducing harmful and hazardous drinking, despite a lack of positive findings in some individual trials. The authors noted that such null effects might be due to a Hawthorne effect, whereby simply joining a trial or having one's drinking recorded influenced the level of drinking. The most commonly assessed drinking outcome was weekly alcohol consumption, with one representative review ${ }^{23}$ suggesting that brief interventions reduced this variable by $38 \mathrm{~g}$ per week (95\% confidence interval [CI] 23 to $54 \mathrm{~g}$ ) for 22 trials of interventions in primary care after one year of follow-up.

This review of reviews ${ }^{22}$ also reported only limited or mixed evidence in terms of the usefulness of brief interventions for women, particularly pregnant women, despite the notion that the antenatal period could represent a time when there is a potent incentive to quit drinking. In addition, for those with more severe drinking problems, systematic reviews have found little support for the effectiveness of brief interventions alone. Among 11 trials of brief interventions in people who were not seeking treatment, the effect on alcohol consumption at three- to six-month follow-up was significantly greater when those with more severe alcohol problems were excluded (aggregate effect size 0.21, $95 \%$ CI 0.14 to 0.27 ) than when they were not excluded $(0.05,95 \%$ CI -0.07 to 0.16$) .{ }^{24}$ Similarly, among 16 randomized controlled trials of alcohol screening and brief intervention in primary care, the 2 trials that did not exclude people with very heavy alcohol use or dependence found no significant effects. ${ }^{25}$

\section{What resources exist to help in administering brief interventions?}

Some nonspecialist providers may find it challenging to address drinking above low-risk levels with their patients (Box 3). The specific challenges include a lack of familiarity with how to identify risky drinking, awkwardness in initiating a conversation about alcohol if it is unrelated to the condition for which treatment is being sought, and perceptions of a lack of expertise and time to intervene. ${ }^{32}$ Other challenges to implementation include a lack of financial incentives; practitioners' feelings about their own 
drinking practices; and the complex social, cultural and moral context concerning what constitutes problematic drinking. ${ }^{33}$ Time constraints may be ameliorated by the emergence of digitally mediated screening and delivery of brief interventions through the Internet or mobile devices ${ }^{34}$ but these are still new and do not yet have a large body of evidence supporting their effectiveness.

A broad range of health care providers without specific addiction expertise are well positioned to identify individuals who drink at levels that are harmful before such patients have even considered changing their alcohol consumption or seeking treatment. Patients are likely to be receptive to a professional with whom they may already have a clinical relationship and whom they trust. In addition, patients may appreciate receiving an intervention in a primary care or hospital setting, which can be less embarrassing, stigmatizing or inconvenient than consulting an addiction specialist or entering a formal alcohol treatment program.

As summarized above (see Box 2), the College of Family Physicians of Canada and the Canadian Centre for Substance Abuse have developed a Web-based resource for Canadian family physicians, nurse practitioners and other health care professionals. ${ }^{7}$ This resource uses Canadian low-risk alcohol guidelines and provides support for screening, brief intervention, referral to specialist treatment and subsequent follow-up. Included is a clinical guide adapted from the US National Institute on Alcohol Abuse and Alcoholism and the British Columbia Ministry of Health, as well as a video training module demonstrating the principles of motivational and nonjudgmental approaches to developing rapport with patients and encouraging them to talk about and make a commitment to change their risky alcohol consumption. The site also includes links to national and international resources, as well as materials for patients, such as guides to low-risk drinking and a workbook to facilitate drinking less. Additional resources are listed in the box "Physician resources."

\section{Unanswered questions}

Although screening and brief interventions for risky or harmful drinking have considerable empiric support, their effectiveness and costeffectiveness for misuse of other drugs remains to be determined. Hampering such efforts is the lack of a practical, brief screening instrument for identifying problematic drug use. Determining the types of clients for whom various intensities and numbers of sessions of brief interventions are appropriate is another question needing more empiric evidence.

\section{References}

1. Giesbrecht N, Stockwell T, Kendall P, et al. Alcohol in Canada: reducing the toll through focused interventions and public health policies. CMAJ 2011;183:450-5.

2. National Alcohol Strategy Working Group. Reducing alcoholrelated harm in Canada: toward a culture of moderation. Recommendations for a national alcohol strategy. Edmonton and Ottawa: Alberta Alcohol and Drug Abuse Commission, Canadian Centre on Substance Abuse and Health Canada; 2007. Available: www.ccsa.ca/Resource\%20Library/ccsa-023876 -2007.pdf (accessed 2014 Feb. 3).

3. Butt P, Beirness D, Gliksman L, et al. Alcohol and health in Canada: a summary of evidence and guidelines for low-risk drinking. Ottawa: Canadian Centre on Substance Abuse; 2011.

4. Stockwell T, Butt P, Beirness D, et al. The basis for Canada's new low-risk drinking guidelines: a relative risk approach to estimating hazardous levels and patterns of alcohol use. Drug Alcohol Rev 2012;31:126-34.

5. Moyer A, Finney JW. Brief interventions for alcohol problems: factors that facilitate implementation. Alcohol Res Health 2004 2005;28:44-50.

6. Breslin FC, Sobell MB, Sobell LC, et al. Toward a stepped care approach to treating problem drinkers: the predictive utility of within-treatment variables and therapist prognostic ratings. Addiction 1997;92:1479-89.

7. Alcohol screening, brief intervention \& referral: helping patients reduce alcohol-related risks. Mississauga and Ottawa (ON): College of Family Physicians of Canada and Canadian Centre on Substance Abuse; 2012. Available: www.sbir-diba.ca (accessed 2014 Jan. 31).

8. Canada's low-risk alcohol drinking guidelines: communicating alcohol-related health risks. Ottawa: Canadian Centre on Substance Abuse; 2013. Available: www.ccsa.ca/Resource\%20 Library/2012-Communicating-Alcohol-Related-Health-Risks-en .pdf (accessed 2014 Feb. 3).

9. Diagnostic and statistical manual of mental disorders. 4th ed., text revision. Washington: American Psychiatric Association; 2000

10. Diagnostic and statistical manual of mental disorders. 5th ed. Washington: American Psychiatric Association; 2013.

11. Alcohol use disorder: a comparison between DSM-IV and DSM-5. NIH Publ. No. 13-7999. Bethesda (MD): National Institute on Alcohol Abuse and Alcoholism; 2013. Available: pubs.niaaa.nih. gov/publications/dsmfactsheet/dsmfact.pdf (accessed 2014 Feb. 22).

12. Bush K, Kivlahan DR, McDonell MB, et al. The AUDIT alcohol consumption questions (AUDIT-C): an effective brief screening test for problem drinking. Arch Intern Med 1998;158:1789-95.

13. Ewing JA. Detecting alcoholism. The CAGE questionnaire. JAMA 1984;252:1905-7.

14. Bradley KA, Kivlahan D, Williams E. Brief approaches to alcohol screening: practical alternatives for primary care. J Gen Intern Med 2009;24:881-3.

15. Lotfipour S, Howard J, Roumani S, et al. Increased detection of alcohol consumption and at-risk drinking with computerized alcohol screening. J Emerg Med 2013;44:861-6.

16. Johnson JA, Woychek A, Vaughan D, et al. Screening for at-risk alcohol use and drug use in an emergency department: integration of screening questions into electronic triage forms achieves high screening rates. Ann Emerg Med 2013;62:262-6.

17. Heather N. Breaking new ground in the study and practice of alcohol brief interventions. Drug Alcohol Rev 2010;29:584-8.

18. Miller WR, Rollnick S. Motivational interviewing. New York: Guilford Press; 1991.

19. Whitlock EP, Green CA, Polen MR, et al. Behavioral counseling interventions in primary care to reduce risky/harmful alcohol use. Rockville (MD): Agency for Healthcare Research and Quality; 2004

20. Watson JM, Crosby H, Dale VM, et al. AESOPS: a randomised controlled trial of the clinical effectiveness and cost-effectiveness of opportunistic screening and stepped care interventions for older hazardous alcohol users in primary care. Health Technol Assess 2013;17:1-158.

21. Fleming MF, Balousek SL, Grossberg PM, et al. Brief physician advice for heavy drinking college students: a randomized controlled trial in college health clinics. J Stud Alcohol Drugs 2010;71:23-31.

22. O'Donnell A, Anderson P, Newbury-Birch D, et al. The impact of brief alcohol interventions in primary healthcare: a systematic review of reviews. Alcohol Alcohol 2014;49:66-78.

23. Kaner EF, Beyer F, Dickinson HO, et al. Effectiveness of brief alcohol interventions in primary care populations. Cochrane Database Syst Rev 2007;(2):CD004148. 
24. Moyer A, Finney JW, Swearingen CE, et al. Brief interventions for alcohol problems: a meta-analytic review of controlled investigations in treatment-seeking and non-treatment-seeking populations. Addiction 2002;97:279-92.

25. Saitz R. Alcohol screening and brief intervention in primary care: absence of evidence for efficacy in people with dependence or very heavy drinking. Drug Alcohol Rev 2010;29:631-40.

26. Ashton M. The motivational hallo. Drug Alcohol Find 2005;13:23-30. Available: http://findings.org.uk/docs/Ashton _M_33.pdf (accessed 2015 Jan. 8).

27. Rohsenow DJ, Monti PM, Martin RA, et al. Motivational enhancement and coping skills training for cocaine abusers: effects on substance use outcomes. Addiction 2004;99:862-74.

28. Pecoraro A, Horton T, Ewen E, et al. Early data from Project Engage: a program to identify and transition medically hospitalized patients into addictions treatment. Addict Sci Clin Pract 2012;7:20.

29. Roozen HG, de Waart R, van der Kroft P. Community reinforcement and family training: an effective option to engage treatment-resistant substance-abusing individuals in treatment. Addiction 2010;105:1729-38.

30. Timko C, DeBenedetti A. A randomized trial of intensive referral to 12-step self-help groups: one-year outcomes. Drug Alcohol Depend 2007;90:270-9.

31. Scott CK, Dennis ML. Results from two randomized clinical trials evaluating the impact of quarterly recovery management checkups with adult chronic substance users. Addiction 2009;104:959-71.

32. Aalto M, Pekuri P, Seppa K. Obstacles to carrying out brief intervention for heavy drinkers in primary health care: a focus group study. Drug Alcohol Rev 2003;22:169-73.

33. O'Donnell A, Wallace P, Kaner E. From efficacy to effectiveness and beyond: What next for brief interventions in primary care? Front Psychiatry 2014;5:113.

34. Struzzo P, Scafato E, McGregor R, et al. A randomised controlled non-inferiority trial of primary care-based facilitated access to an alcohol reduction website (EFAR-FVG): the study protocol. BMJ Open 2013;3. pii:e002304.

Affiliations: Stony Brook University (Moyer), Stony Brook, NY; Center for Innovation to Implementation (Finney), VA Palo Alto Health Care System, Menlo Park, Calif.
Contributors: Both authors drafted the article or revised it critically for important intellectual content, gave final approval of the version to be published and agree to act as guarantors of the work.

\section{Physician resources}

- Alcohol Screening, Brief Intervention \& Referral (SBIR), a three-step clinical Web resource for managing patients' alcohol consumption (College of Family Physicians of Canada and Canadian Centre on Substance Abuse): www.sbir-diba.ca

- College Health Intervention Projects: Creating a Healthier Lifestyle (University of Wisconsin, Department of Family Medicine): www.fammed.wisc.edu/files/webfm-uploads /documents/research/workbook_chips_v6.pdf

- Alcohol Screening and Brief Intervention for Youth: A Practitioner's Guide (US Department of Health and Human Services, National Institutes of Health, National Institute on Alcohol Abuse and Alcoholism): http://pubs .niaaa.nih.gov/publications/Practitioner/ YouthGuide/YouthGuide.pdf

- Helping Patients Who Drink Too Much: A Clinician's Guide (US Department of Health and Human Services, National Institutes of Health, National Institute on Alcohol Abuse and Alcoholism): http://pubs.niaaa.nih.gov /publications/Practitioner/CliniciansGuide2005/ guide.pdf

\section{$\mathbf{R E P R}$

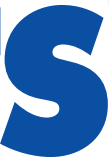 \\ We have partnered with Sheridan Press!}

To purchase commercial article reprints and e-prints or to request a quote, please contact

Matt Neiderer

Content Sales Sheridan Content Services
$800635-7181 \times 8265$ matt.neiderer@sheridan.com 